\title{
Priming T2 in a visual and auditory attentional blink task
}

\author{
Thomas KoelewiJn and Erik Van der Burg \\ Vrije Universiteit Amsterdam, Amsterdam, The Netherlands \\ Adelbert BRONKHORST \\ Vrije Universiteit Amsterdam, Amsterdam, The Netherlands \\ and TNO Human Factors, Soesterberg, The Netherlands \\ AND \\ JAN THEEUWES \\ Vrije Universiteit Amsterdam, Amsterdam, The Netherlands
}

\begin{abstract}
Participants performed an attentional blink (AB) task including digits as targets and letters as distractors within the visual and auditory domains. Prior to the rapid serial visual presentation, a visual or auditory prime was presented in the form of a digit that was identical to the second target (T2) on 50\% of the trials. In addition to the "classic" AB effect, an overall drop in performance on T2 was observed for the trials on which the stream was preceded by an identical prime from the same modality. No cross-modal priming was evident, suggesting that the observed inhibitory priming effects are modality specific. We argue that the present findings represent a special type of negative priming operating at a low feature level.
\end{abstract}

Capacity limitations in the visual system become evident when a vast amount of information needs to be processed within a limited period of time. A classic example of such a capacity limitation is the attentional blink (AB) deficit (Broadbent \& Broadbent, 1987; Raymond, Shapiro, \& Arnell, 1992). An AB occurs when people have to report two target items (e.g., words or single characters) presented among distractors in a rapid serial visual presentation (RSVP), in which items are presented in succession at a high rate (e.g., 10 items per second). People are accurate in reporting the first target (T1) but often fail to report the second target (T2). The $\mathrm{AB}$ is most severe when $\mathrm{T} 1$ and $\mathrm{T} 2$ are presented close together in time (within $200-500 \mathrm{msec}$ ) but gradually disappears as the time period between the targets becomes longer.

One of the early models explaining the $\mathrm{AB}$ effect is the two-stage model of Chun and Potter (1995). As its name implies, this model divides target detection into two stages: In the first stage, relevant features of the target are detected, and in the second, the target is consolidated into short-term memory (STM). The model states that the $\mathrm{AB}$ deficit is based on a capacity limitation that occurs during consolidation of T2 into STM (Jolicœur \& Dell'Acqua, 1998). The AB occurs because resources used during consolidation of $\mathrm{T} 1$ are not available at the time when consolidating T2 is necessary. This results in a bottleneck in the transfer of sensory codes to STM.
According to the two-stage model, consolidation is necessary for reporting T2. To get a better understanding of the process underlying the $\mathrm{AB}$, it is important to know whether or not prior knowledge already consolidated into STM has an influence on the AB. In other words, what happens to $\mathrm{T} 2$ performance when an item identical to $\mathrm{T} 2$ has already been shown and consolidated into STM prior to the presentation of T2? The classic study by Jacoby and Dallas (1981) showed that identification of an item (e.g., a word) improves as a result of prior exposure to a similar or identical item. This effect is called repetition priming, and on the basis of the effect repetition priming has on performance, an improvement of T2 performance can be expected.

However, Akyürek and Hommel (2005) found an overall performance drop on $\mathrm{T} 2$ in an $\mathrm{AB}$ task when participants held in memory characters from the same class as T2. In this paradigm, participants had to memorize in each trial a number of characters (letters, digits, or symbols), displayed prior to the RSVP stream, which they had to report back afterward. Their performance on the AB task became worse as the STM load became higher and as the STM content became more related to the targets (letters, digits, or symbols) in the AB task. Interestingly, the drop in performance did not interact with the $\mathrm{AB}$ and was constant over the lag condition. Another study (Nieuwenstein, Johnson, Kanai, \& Martens, 2007) showed a similar drop in T2 performance when an STM set contained an item identi-

T. Koelewijn, t.koelewijn@psy.vu.nl 
cal to T2, as compared with an STM set without identical items. Both studies showed that when an item identical to or from the same class as T2 is already consolidated in STM, performance on T2 drops. Thus, performance on T2 seems to suffer because of competition between related items already stored in STM (Akyürek \& Hommel, 2005), whereas the $\mathrm{AB}$ deficit itself is assumed to be the result of capacity limitations during the consolidation of items into STM (Jolicœur \& Dell'Acqua, 1998). Nieuwenstein et al. explained this effect as a failure in attributing the same item information to both the STM and AB tasks. They referred to the additional failure in reporting $\mathrm{T} 2$ when this item is already coupled to a different task - in this case, an STM task-as "cross-talk repetition amnesia." Both Akyürek and Hommel and Nieuwenstein et al. concluded that the additional drop in $\mathrm{T} 2$ performance does not occur during the consolidation stage, but instead seems to reflect interference in STM and to be based on a different process than the $\mathrm{AB}$.

The cross-talk repetition amnesia hypothesized by Nieuwenstein et al. (2007) is in line with the episodic retrieval model (DeSchepper \& Treisman, 1996; Neill \& Mathis, 1998; Neill, Valdes, Terry, \& Gorfein, 1992), which is used to explain a process called negative priming (NP). The label $N P$ is broadly used for perceptual inhibitory processes (Neill \& Mathis, 1998; Rothermund, Wentura, \& De Houwer, 2005; Tipper, 1985; Wood \& Milliken, 1998). The classical NP paradigm (Tipper, 1985) consists of prime and probe trials, each containing a target and a distractor item. When a distractor item that needs to be ignored in the prime trial becomes a target item in a subsequent probe trial, the response to this target tends to be slower. The inhibition model of NP (Houghton, Tipper, Weaver, \& Shore, 1996; Tipper, 1985; Tipper, Weaver, Cameron, Brehaut, $\&$ Bastedo, 1991) explains this effect by inhibition of a to-be-ignored item. When the inhibited item becomes a target, the activation threshold of the item is higher than that of uninhibited targets. The episodic retrieval theory of NP (DeSchepper \& Treisman, 1996; Neill \& Mathis, 1998; Neill et al., 1992), on the other hand, states that the representation of an item is stored along with an "action tag" indicating what to do with the item. This action tag could involve the coupling to a task, as suggested by crosstalk repetition amnesia. In the case of NP, a distractor item stored with a no-response tag creates conflict when it becomes a target item to which participants have to respond.

In the present study, participants were presented with an RSVP stream of letters containing two digits as targets. Prior to the RSVP stream, a prime was presented that was either identical or not identical to $\mathrm{T} 2$, but which always belonged to the same class (digits) as the target. Instead of an additional memory task, as was used by Akyürek and Hommel (2005), a single prime was used. The question we addressed was whether a performance reduction would show up, similar to the one reported in experiments that used an additional memory task, or whether a positive enhancement effect would show up, due to repetition priming. To anticipate the results, our Experiment 1 showed an inhibitory effect on T2 performance when the target was primed by a physically identical item, which is in line with previous work (Akyürek
\& Hommel, 2005; Nieuwenstein et al., 2007). Three additional experiments were conducted to investigate whether this effect could be explained in terms of NP.

A second objective of this study was to determine whether this effect on $\mathrm{T} 2$ performance is restricted to the visual domain. On the basis of the previous priming (Graf, Shimamura, \& Squire, 1985) and NP (Buchner, Zabal, \& Mayr, 2003) literature, it is quite feasible that similar effects exist in auditory, and even cross-modal, conditions. To answer this question, two additional experiments were conducted using an auditory or a visual serial stream preceded by an auditory prime.

\section{EXPERIMENT 1 Visual-Visual Priming}

In Experiment 1, participants were presented with an RSVP stream containing two target digits among distractor letters. Prior to the RSVP stream, a prime was presented that was either identical or not identical to T2. The participants had to report whether the target digits presented within the RSVP stream were odd or even.

\section{Method}

Participants. Twelve students of Vrije Universiteit Amsterdam (9 female, 3 male; mean age 22.3 years, age range 19-33) took part in the experiment. All had normal or corrected-to-normal vision. The participants were informed beforehand about the experimental procedure and were naive as to the purpose of the experiment.

Design and Stimuli. This experiment had a $2 \times 4$ design with the factors prime (prime $\neq \mathrm{T} 2$, prime $=\mathrm{T} 2$ ) and lag $(1,2,3$, or 8 ). The RSVP stream contained 20 elements, each of which was presented for $16 \mathrm{msec}$, followed by an interstimulus interval (ISI) of $80 \mathrm{msec}$. T1 was presented at Position 7, 8, or 9 in the RSVP stream, and T2 was positioned at a lag of 1, 2, 3, or 8 after T1. All T1 and T2 position combinations occurred equally often, in a random order. Prior to the RSVP stream, a prime was displayed for $1.5 \mathrm{sec}$, followed by a fixation cross for $200 \mathrm{msec}$, as shown in Figure 1. The prime was displayed in all trials; it was identical to T2 on $50 \%$ of the trials and was never identical to T1. The digits $1-9$ (5 excluded) were used for the prime, T1, and T2, and the distractors were capital letters of the alphabet (with the letters $I$ and $X$ excluded). All characters were displayed at the center of the screen in dark gray, 48-point Geneva font $\left(0.63 \mathrm{~cd} / \mathrm{m}^{2}, 1.4^{\circ}\right.$ width, $1.6^{\circ}$ height $)$ on a gray $\left(9.34 \mathrm{~cd} / \mathrm{m}^{2}\right)$ background. During practice, all characters were displayed in black in order to familiarize participants with the task.

Apparatus and Procedure. The participants were seated in a dimly lit cabin approximately $80 \mathrm{~cm}$ from a computer screen (17-in., $120-\mathrm{Hz}$ ). The experiment was run in E-Prime 1.1 (SP3). The task instructions were presented onscreen, after which the participants started with a practice block of 48 trials. The participants were instructed to look at the prime digit, but also that the digit was irrelevant for the task and did not need to be responded to. After each block, the participants received feedback on their overall performance. The experiment consisted of six blocks containing 48 trials each. The participants had to respond (unspeeded) to T1 and T2 sequentially, by pressing the "o" key for odd or "e" key for even on a QWERTY keyboard.

\section{Results}

For all analyses, a significance level of $p<.05$ was used, and $M S_{\mathrm{e}}$ and $p$ values were Greenhouse-Geisser adjusted when required. Two separate repeated measurements ANOVAs were conducted for performance on $\mathrm{T} 1$ and for performance on $\mathrm{T} 2$, given that $\mathrm{T} 1$ was correctly identified. 


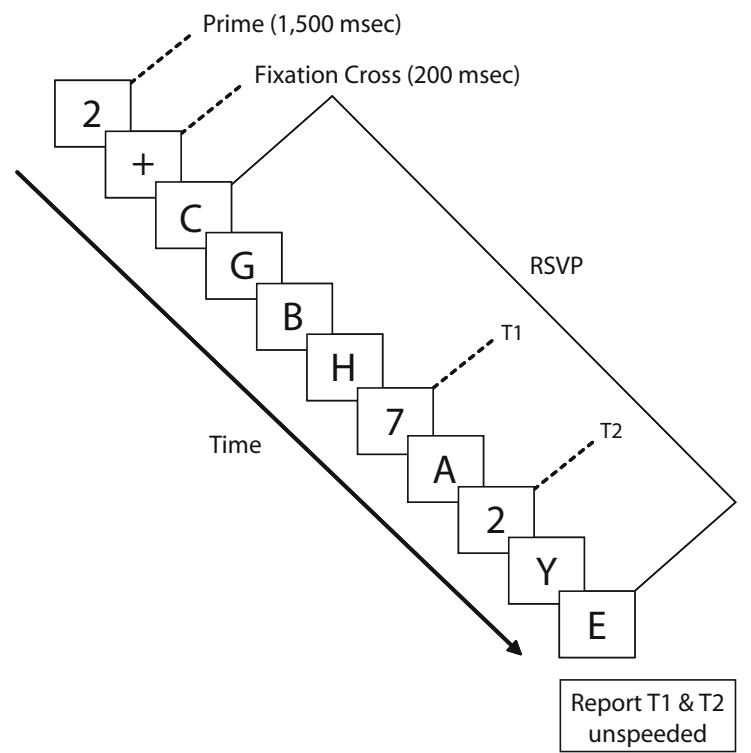

Figure 1. Schematic representation of the paradigm used. Participants received a rapid serial visual presentation (RSVP). The task was to identify the two targets (T1 and T2), which were digits, and to report, unspeeded and in order of appearance, whether they were odd or even. Prior to the RSVP stream, a prime was presented for $1.5 \mathrm{sec}$. In $50 \%$ of the trials, the prime was identical to $\mathrm{T} 2$.

Prime and lag were within-subjects variables. Figure 2 shows the mean percentages correct for $\mathrm{T} 2$ given that $\mathrm{T} 1$ was correctly identified, as a function of lag and prime.

T1 accuracy. Overall, performance was $91 \%$ correct. For the prime $=\mathrm{T} 2$ condition, the percentage-correct scores for T1 for lags $1,2,3$, and 8 were $88 \%, 91 \%, 92 \%$, and $96 \%$, respectively. In the same order, the results for the prime $\neq$ $\mathrm{T} 2$ condition were $83 \%, 93 \%, 92 \%$, and $95 \%$. The main effect of prime failed to reach significance $(F<1)$, but performance did vary with $\operatorname{lag}\left[F(3,33)=10.523, M S_{\mathrm{e}}=.006\right.$,

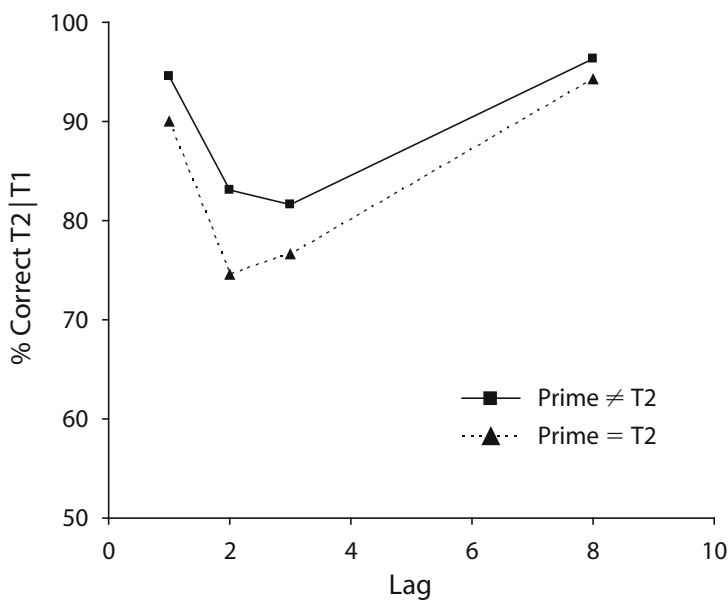

Figure 2. Results of Experiment 1, in percentages correct, for $\mathrm{T} 2$ given $\mathrm{T} 1$ correct $(\mathrm{T} 2 \mid \mathrm{T} 1)$ as a function of lag and prime. $p<.005$ ], and the two-way interaction between prime and lag was significant $\left[F(3,33)=3.401, M S_{\mathrm{e}}=.003, p<.05\right]$. The interaction was further analyzed by pairwise $t$ tests between the prime conditions for each lag (1-3 and 8), which only revealed a significant effect for lag $1(p<.05)$. The main effect of lag was also further analyzed by pairwise $t$ tests between lags. These results only showed a significant effect for lag 1 relative to lag $2(p<.05)$.

T2 accuracy. Performance varied with lag $[F(3,33)=$ $\left.16.357, M S_{\mathrm{e}}=.023, p<.001\right]$. There was also a significant effect of prime $\left[F(1,11)=12.368, M S_{\mathrm{e}}=.005, p<\right.$ $.01]$, resulting in an overall drop in performance when T2 was primed (84\%) as compared with when it was not primed $(89 \%)$. The two-way interaction between prime and lag failed to reach significance $[F(3,33)=1.525$, $\left.M S_{\mathrm{e}}=.004, p=.236\right]$.

\section{Discussion}

Priming of T2 did not affect T1 performance, with the exception of at the first lag. The drop in performance on T1 for lag 1 is similar to the effect reported by Akyürek and Hommel (2005), as well as to that reported by Potter, Staub, and O'Connor (2002). The researchers explained this effect in terms of competition between two succeeding targets when they are selected from the same set of characters (digits). This competition seems to be strongest when $\mathrm{T} 2$ is not identical to the prime, as shown by the observed interaction. Another explanation for this effect could be that participants remembered $\mathrm{T} 1$ and $\mathrm{T} 2$ in the incorrect order. At the end of the trial, participants had to report whether the two targets (T1 and T2) were odd or even. This had to be done unspeeded and in the correct order. Thus, not only the targets themselves had to be remembered, but also the order of their appearance. When targets are presented close in time, as is the case for lag 1, and both characters are from the same character class, an order judgment can become difficult, resulting in a drop in $\mathrm{T} 1$ performance on lag 1. Note that this effect is not apparent in the scores, since only the scores of T2 given T1 correctly identified are shown.

The typical U-shaped drop in T2 performance from lag 1 to lag 8 shown in Figure 2 reflects a standard $A B$ effect (Raymond et al., 1992). The results further show an overall inhibitory effect of prime on T2 performance and no interaction between prime and lag. These results are in line with earlier work (Akyürek \& Hommel, 2005; Nieuwenstein et al., 2007) and show that when an item identical to T2 is previously consolidated, it becomes harder for participants to correctly identify $\mathrm{T} 2$.

In this experiment, participants were instructed to look at the prime but were not required to actively maintain it. Unlike in a typical NP paradigm, in which participants have to ignore the prime in order to make the appropriate response, in the present experiment observers were asked to attend to the prime but did not have to give an overt response. However, even though we did not instruct participants to do so, we cannot rule out that the participants actively suppressed the prime as a strategy to enhance their task performance. If participants did actively suppress the prime, this would be consistent with the idea that the drop in performance during the $\mathrm{AB}$ task is the result of inhibition. 
According to the inhibition model, if participants actively maintained a prime, one would not expect a drop in performance for T2 (since there would be no need for inhibition); if anything, on the basis of Tipper's (1985) results, one would expect a performance benefit for $\mathrm{T} 2$. Experiment 2 was basically a replication of Experiment 1, but now we ensured that participants actively maintained the prime in STM.

\section{EXPERIMENT 2 Memorizing the Prime}

In this experiment, participants were instructed to memorize the prime instead of just attending to it. To make sure that they followed the instructions, we added a few so-called prime recall trials. In these trials, participants had to report the identity of the prime after presentation of the RSVP stream. Because the prime was now actively maintained in STM, we expected enhanced $\mathrm{T} 2$ performance when the prime and $\mathrm{T} 2$ were identical (Tipper, 1985).

\section{Method}

The present experiment was identical to Experiment 1, except that participants were instructed to memorize the prime presented in each trial. Additional prime recall trials were included, which constituted $20 \%$ of the trials. These trials were identical to the other trials, with the exception that a different response had to be given: Instead of reporting the targets, a number word (e.g., the word eight) appeared onscreen, and participants were asked to indicate by pressing " $\mathrm{j}$ " for yes and " $\mathrm{n}$ " for no whether the number word displayed was the same as or different from the prime digit kept in memory. Twelve new students ( 6 female, 6 male; mean age 23.3 years, age range 17-37) participated in the experiment.

\section{Results}

On average, participants scored $98 \%$ correct on the prime recall trials. This provides a strong indication that they actively observed the prime and maintained it in STM during a trial.

T1 accuracy. T1 was correctly identified on $87 \%$ of the trials. For the prime $=\mathrm{T} 2$ condition, $\mathrm{T} 1$ performance at lags $1,2,3$, and 8 was $80 \%, 90 \%, 88 \%$, and $92 \%$, respectively. In the same order, the results for the prime $\neq \mathrm{T} 2$ condition were $76 \%, 88 \%, 91 \%$, and $93 \%$. There was no significant effect of prime on T1 performance $(F<1)$, but performance did vary significantly with lag $[F(3,33)=$ $\left.9.336, M S_{\mathrm{e}}=.022, p<.004\right]$, and there was no significant interaction between prime and lag $[F(3,33)=1.073$, $\left.M S_{\mathrm{e}}=.005, p=.363\right]$.

T2 accuracy. The average scores for each condition are shown in Figure 3. T2 given T1 correct varied with lag $\left[F(3,33)=20.381, M S_{\mathrm{e}}=.029, p<.001\right]$, indicating an $\mathrm{AB}$ effect. There was a significant main effect of prime on $\mathrm{T} 2$ performance $\left[F(1,11)=9.308, M S_{\mathrm{e}}=.007\right.$, $p<.05$ ] resulting in poorer performance when T2 was primed $(78 \%)$ than when it was not primed $(83 \%)$. The two-way interaction between prime and lag was significant $\left[F(3,33)=4.299, M S_{\mathrm{e}}=.008, p<.05\right]$; a further analysis by two-tailed $t$ tests for each lag $(1,2,3$, and 8$)$ only showed a significant effect for lag $2(p<.05)$.

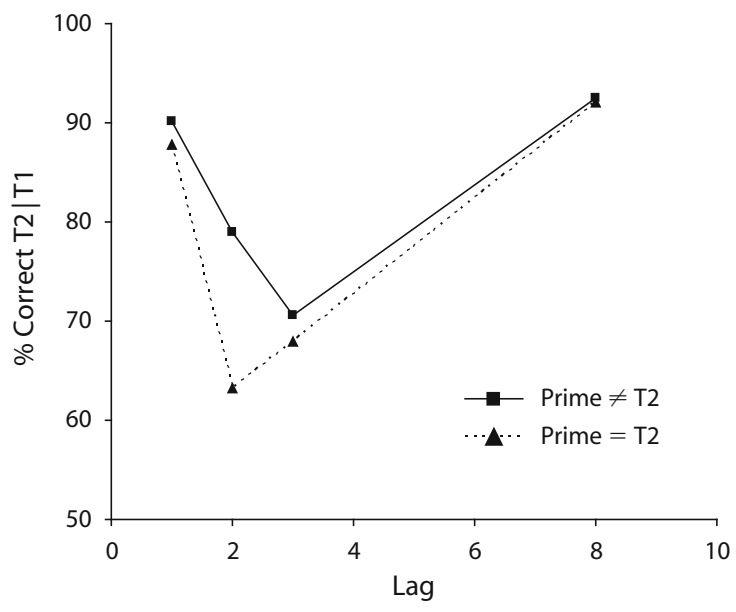

Figure 3. Results of Experiment 2, in percentages correct, for $\mathrm{T} 2$ given $\mathrm{T} 1$ correct $(\mathrm{T} 2 \mid \mathrm{T} 1)$ as a function of lag and prime.

Comparing overall T2 $\mid \mathrm{T} 1$ performance between Experiments 1 and 2, by means of one-sided independentsamples $t$ tests, revealed a significant $(p=.0265)$ drop in performance for Experiment 2 relative to Experiment 1.

\section{Discussion}

The present experiment replicated and extended the findings of Experiment 1 by showing an inhibitory effect of prime on T2 performance, even when it was ensured that the prime was stored in STM. Thus, contrary to the predictions of the inhibition model of NP (Houghton et al., 1996; Tipper et al., 1991), in the present paradigm actively maintaining the prime did not lead to facilitation. Note that these results are in line with earlier studies that have shown a similar drop in T2 performance when items related to T2 were actively maintained in STM (Akyürek \& Hommel, 2005; Nieuwenstein et al., 2007). However, in contrast to Experiment 1, the present results showed an interaction between prime and lag, indicating a clear priming effect for lag 2 but not for the other lags.

It is likely that this interaction was due to the fact that the memory task had an overall detrimental effect on performance. Regardless of whether the prime matched T2 or not, performance at lags 2 and 3 was much worse in Experiment 2 than in Experiment 1. It is feasible that in Experiment 2 the effect of the prime differed over lags because the overall performance decrement was close to ceiling. Therefore, the interaction may have been due to the fact that the memory task caused a strong performance decrement that was only marginally affected by the addition of the prime. Regardless of this interaction, the most important outcome of the present experiment was the main effect of the prime on T2 performance that was opposite to what the inhibition model would predict when the prime did not have to be inhibited.

All in all, the present findings suggest that the negative effect on T2 performance may be related to NP, although this would not fit the inhibition model. If this is indeed the case, the negative effect then need not depend on whether 
the prime is physically identical to the target (for a review, see Neill \& Mathis, 1998). For instance, NP could also occur when using either pictures or words that are semantically related to the target (Tipper, 1985; Yee, 1991). If the effect observed in Experiments 1 and 2 is based on NP, similar effects should also be observed when using a semantically related rather than an identical prime. To test this assumption, a third experiment was conducted in which, instead of a digit (e.g., 8), a number word (e.g., eight) was presented as the prime.

\section{EXPERIMENT 3 Semantic Priming}

In this experiment, participants observed a prime in the form of a number word that was either the same number as the target (e.g., prime eight, target 8) or a different number (e.g., prime eight, target 2). T1 and T2 were still presented as single digits. In contrast to Experiment 1, the prime and T2 were not identical but shared the same semantics. This experiment allowed us to investigate the influence of semantic priming on $\mathrm{T} 2$ performance.

\section{Method}

The present experiment was identical to Experiment 1, except that a semantic prime was presented as a number word in Dutch (één, twee, drie, vier, zes, zeven, acht, and negen). Sixteen new students (10 female, 6 male; mean age 20.2 years, age range 17-31) participated in the experiment.

\section{Results}

T1 accuracy. T1 was correctly identified on $94 \%$ of the trials. For the prime $=\mathrm{T} 2$ condition, the performance on lags $1,2,3$, and 8 was $87 \%, 94 \%, 97 \%$, and $97 \%$, respectively. In the same order, the results for the prime $\neq \mathrm{T} 2$ condition were $86 \%, 96 \%, 98 \%$, and $98 \%$. There was no significant effect of prime on $\mathrm{T} 1$ performance $[F(1,15)=$ $\left.1.259, M S_{\mathrm{e}}=.001, p=.280\right]$, but performance did vary significantly across lags $\left[F(3,45)=25.833, M S_{\mathrm{e}}=.007\right.$, $p<.001]$. Further analysis by means of a two-tailed pairedsamples $t$ test showed a significant performance drop on lag 1 as compared with lag $2[t(15)=4.612, p<.001]$ and on lag 2 as compared with lag $3[t(15)=3.381, p<.005]$. No significant two-way interaction between prime and lag was found $\left[F(3,45)=1.142, M S_{\mathrm{e}}=.001, p=.338\right]$.

T2 accuracy. The average scores for the individual conditions are shown in Figure 4. The performance on T2 given T1 correct varied with lag $[F(3,45)=13.595$, $\left.M S_{\mathrm{e}}=.013, p<.005\right]$, indicating an AB effect. For T2, there was no significant main effect of prime $(F<1)$ or two-way interaction between prime and lag $(F<1)$.

\section{Discussion}

When the target was primed semantically, no effect on T2 performance was found. These results differ from those of Experiments 1 and 2, in which performance on a primed T2 dropped relative to performance on a nonprimed T2. For an inhibitory effect to occur (as shown in Experiment 1 ), it seems necessary that the prime be physically identical to the target. Therefore, the present results are not in line with the classic NP explanation referred to as the

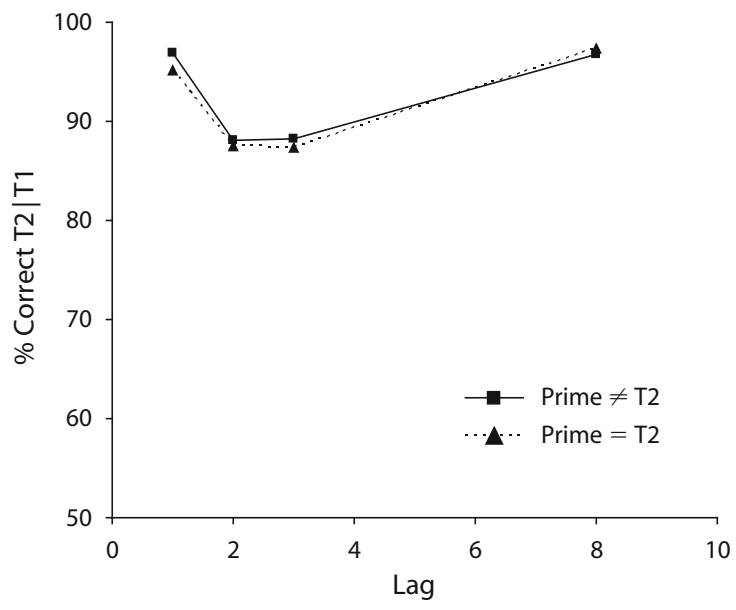

Figure 4. Results of Experiment 3, in percentages correct, for $\mathrm{T} 2$ given $\mathrm{T} 1$ correct $(\mathrm{T} 2 \mid \mathrm{T} 1)$ as a function of lag and prime.

inhibition model, as developed by Tipper and colleagues (Houghton et al., 1996; Tipper, 1985; Tipper et al., 1991). However, before excluding NP as an explanation for the performance decrement, a close comparison between the NP and present paradigms is needed.

The paradigm here differs from the classic NP paradigm of Tipper (1985). Typically, the NP paradigm consists of prime and probe trials, each containing a target and a distractor item. NP occurs when an ignored distractor item in the prime trial becomes a target in the probe trial. Still, there are also similarities between the two paradigms. First of all, in both paradigms two targets are presented. This means that T1 in the RSVP stream can be seen as the target in the prime trial and T2 as the target in the probe trial. Second, in both paradigms distractors are present. In addition to the distractors presented in the RSVP stream surrounding T2, the prime presented prior to the RSVP stream could function as a distractor for T1. Note that, in the present experiment, targets and distractors were not presented simultaneously; however, a study by Neill and Mathis (1998) showed that this has no consequences for NP. It could be that the interaction between the prime and T1, both from the same character class, causes the inhibition effect on T2 performance. When $\mathrm{T} 1$ and the prime are less related (e.g., are from different character classes), there should be less competition between them, and therefore no reason for the prime to be inhibited.

\section{EXPERIMENT 4 Different Character Class Used for T1}

In Experiments 1 and 2, the prime, T1, and T2 were all digits. Because all were from the same class of stimuli, the prime could act as a distractor item for T1. To determine whether this was indeed the case, T1 in this experiment was taken from a different character class from that of the prime and T2. If it is true that the prime is suppressed because it competes with T1, we would expect the NP effect to disappear when $\mathrm{T} 1$ is from a different character class than are the prime and $\mathrm{T} 2$. 


\section{Method}

Experiment 4 was similar to Experiment 1, except that instead of digits, the symbols "\#" and "\%" were used for T1. Participants had to indicate which symbol they had seen by pressing the " $\mathrm{z}$ " key for the "\#" symbol and the " $m$ " key for the "\%" symbol. For T2, the same digits were shown as in the previous experiments, and again participants had to indicate whether the digit was odd or even. During a pilot study, it became clear that this change made the task much easier and that performance on $\mathrm{T} 1$ and $\mathrm{T} 2$ might reach ceiling. Therefore, the ISI between the items in the RSVP stream was reduced from $80 \mathrm{msec}$ to $40 \mathrm{msec}$, which made the task equally difficult as the tasks in Experiments 1 and 2. Eight new students ( 6 female, 2 male; mean age 20.8 years, age range $18-25$ ) participated in the experiment.

\section{Results}

T1 accuracy. T1 was correctly identified on $95 \%$ of the trials. For the prime $=\mathrm{T} 2$ condition, the performance at lags $1,2,3$, and 8 was $97 \%, 95 \%, 93 \%$, and $95 \%$, respectively. In the same order, the results for the prime $\neq \mathrm{T} 2$ condition were $97 \%, 94 \%, 95 \%$, and $96 \%$. There was no significant effect of the prime on $\mathrm{T} 1$ performance $(F<1)$, but performance did vary significantly with lag $\left[F(3,21)=4.441, M S_{\mathrm{e}}=\right.$ $.001, p<.05]$. Further analysis by means of a two-tailed paired-samples $t$ test showed a significant performance drop at lag 3 as compared with lag $8[t(7)=-2.728, p<.05]$. No significant two-way interaction was found between prime and lag $\left[F(3,21)=1.359, M S_{\mathrm{e}}=.001, p=.287\right]$.

T2 accuracy. The average scores for the individual conditions are shown in Figure 5. T2 given a correct T1 varied with lag $\left[F(3,21)=11.133, M S_{\mathrm{e}}=.006, p<\right.$ .005]. Prime had no significant effect on T2 performance $(F<1)$, and the two-way interaction between prime and lag was not significant $(F<1)$.

\section{Discussion}

In contrast to what was found in Experiments 1 and 2, priming of T2 now had no effect on T2 performance. Changing T1 into a symbol apparently made the prime no longer a distractor item for T1, and therefore no additional inhibitory effect was observed. This is in line with the idea that NP occurs when a distractor item is suppressed in favor of the target item in the prime trial (Houghton et al., 1996; Tipper, 1985; Tipper et al., 1991).

Displaying T1 as a symbol did not affect the AB, as shown by the main effect of lag that was still present. However, no typical $U$ shape in the data, a characteristic of the $\mathrm{AB}$, was found. One reason for this may be that the $U$ shape of the $\mathrm{AB}$ is based on the time between $\mathrm{T} 1$ and $\mathrm{T} 2$, and not on the number of lags between the two targets (Martens, Munneke, Smid, \& Johnson, 2006). After we sped up the RSVP stream, at lag 8 only $448 \mathrm{msec}$ had passed, instead of the $768 \mathrm{msec}$ in Experiments 1 and 2. Obviously, after $448 \mathrm{msec}$ one would still expect an AB effect.

A minor point of discussion in Experiment 1 was the drop in T1 performance at lag 1. We suggested that this effect could be explained by the fact that the task required a correct order judgment of the targets, which became difficult when targets were presented as close together in time as on lag 1 . In the present experiment, no correct order judgment was necessary, because the participants simply knew that a target symbol was presented before a target digit.

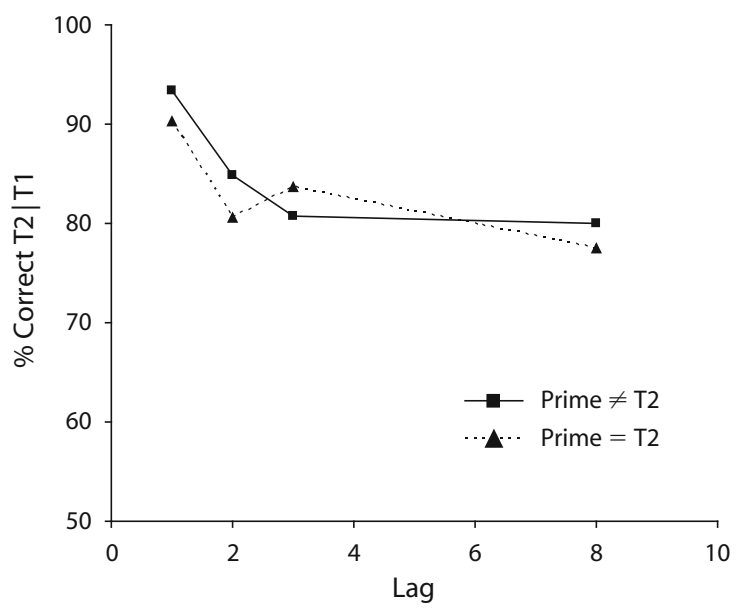

Figure 5. Results of Experiment 4, in percentages correct, for $\mathrm{T} 2$ given $\mathrm{T} 1$ correct $(\mathrm{T} 2 \mid \mathrm{T} 1)$ as a function of lag and prime.

Even though the effect of lag remained significant, post hoc analyses revealed no significant drop in T1 performance at lag 1 , which is consistent with the explanation above.

A second objective of the present study was to determine whether the additional inhibitory effect on T2 performance is restricted to the visual domain. In the following experiment, we investigated whether an auditory prime influences the performance for an auditory $\mathrm{T} 2$. Such an effect might be expected, because Buchner et al. (2003) found NP effects in the auditory domain when participants had to discriminate between the sounds of animals or of musical instruments.

\section{EXPERIMENT 5 Auditory-Auditory Priming}

The additional inhibition effect on T2 performance, as shown in Experiments 1 and 2, seems to happen at an early stage of visual processing. This raises the question of whether this effect is specific to the visual domain. To address this issue, participants were presented with a rapid serial auditory presentation (RSAP) stream, comparable to the RSVP stream used in all previous experiments, that was preceded by an auditory prime. The task and design were similar to those of Experiment 1.

\section{Method}

For this experiment, auditory stimuli were used in the form of spoken letters (distractors) and numbers (targets and prime). The letters used as distractors in the RSAP stream were $B, C, D, F$, $G, H, J, K, L, M, N, O, R, U, V, W$, and $X$. For the prime and targets, the numbers $1,2,3$, and 4 were used. All letters and numbers were spoken in Dutch by a male voice and were compressed to a duration of $90 \mathrm{msec}$. Identical auditory stimuli were used for both primes and targets, and prime onset was time-locked 1,500 msec before the onset of the fixation cross. All vocals were digitally recorded and edited with a 16-bit resolution and 44-kHz sampling rate using Cool Edit Pro 2.1 software. During recording, voice inflections were kept to a minimum. The amplitudes of all samples were manually normalized, and time compression was performed by means of a time-stretching routine that manipulated duration without altering pitch. 
To make sure that the participants perceived the edited spoken digits correctly, a pilot study was conducted with 4 participants. These participants were presented with an RSAP stream similar to the one used in the experiment, but that contained only one target digit; no prime was presented prior to the stream. The participants then had to give a speeded response by pressing the corresponding number on the keyboard. In this pilot study, all numbers from 1 to 9 were tested; on the basis of the results, the numbers 1 to 4 were selected as the best candidates for the experiment. Nine new students ( 5 female, 4 male; mean age 21.5 years, age range 18-30) participated in the experiment. All had normal hearing and vision.

\section{Results}

The data of 1 participant were excluded from further analysis because of failure to comply with the task instructions.

T1 accuracy. T1 was correctly identified on $84 \%$ of the trials. For the prime $=\mathrm{T} 2$ condition, the performance on lags $1,2,3$, and 8 was $83 \%, 85 \%, 86 \%$, and $85 \%$, respectively. In the same order, the results for the prime $\neq$ T2 condition were $81 \%, 81 \%, 84 \%$, and $87 \%$. There was no significant effect of prime $\left[F(1,7)=2.418, M S_{\mathrm{e}}=\right.$ $.002, p=.164]$ or $\operatorname{lag}\left[F(3,21)=2.253, M S_{\mathrm{e}}=.005, p=\right.$ $.146]$ on $\mathrm{T} 1$ performance, nor was there a two-way interaction between prime and lag $(F<1)$.

T2 accuracy. The average scores for the individual conditions are shown in Figure 6. T2 given T1 correct did not vary across lags $(F<1)$, indicating no $\mathrm{AB}$ effect. There was a significant effect of prime on $\mathrm{T} 2$ performance $\left[F(1,7)=8.449, M S_{\mathrm{e}}=.002, p<.05\right]$, resulting in an overall drop in performance when T2 was primed (86\%) as compared with when it was not primed (90\%). The twoway interaction between prime and lag was not significant $(F<1)$.

\section{Discussion}

In this experiment, no $\mathrm{AB}$ effect was found. This is in line with the results of earlier studies (e.g., Arnell \& Jenkins, 2004; Potter, Chun, Banks, \& Muckenhoupt, 1998) that have shown that auditory ABs can occur, but not when number targets among letter distractors need to be reported. Nevertheless, we did find an inhibitory effect of the prime on T2 performance, suggesting that the NP effect is not specific to the visual modality. The fact that it occurs in the absence of an $\mathrm{AB}$ underlines the idea that $\mathrm{NP}$ taps into different resources than does the AB.

Experiments 1,2, and 5 show inhibitory effects on $\mathrm{T} 2$ performance in both the visual and auditory domains. It is well known (see, e.g., Arnell \& Jolicœur, 1999; Spence \& Driver, 1997) that auditory input can have an effect on processing visual information. Furthermore, Buchner et al. (2003) have demonstrated NP for a prime and a target presented in different modalities. A relevant question, therefore, is whether similar cross-modal inhibitory effects will show up when we use a cross-modal variant of our paradigm.

\section{EXPERIMENT 6 Auditory-Visual Priming}

Experiment 6 tests whether the inhibitory effects shown in Experiments 1 and 2 (visual) and 5 (auditory) hold in a cross-modal setting in which an RSVP stream is pre-

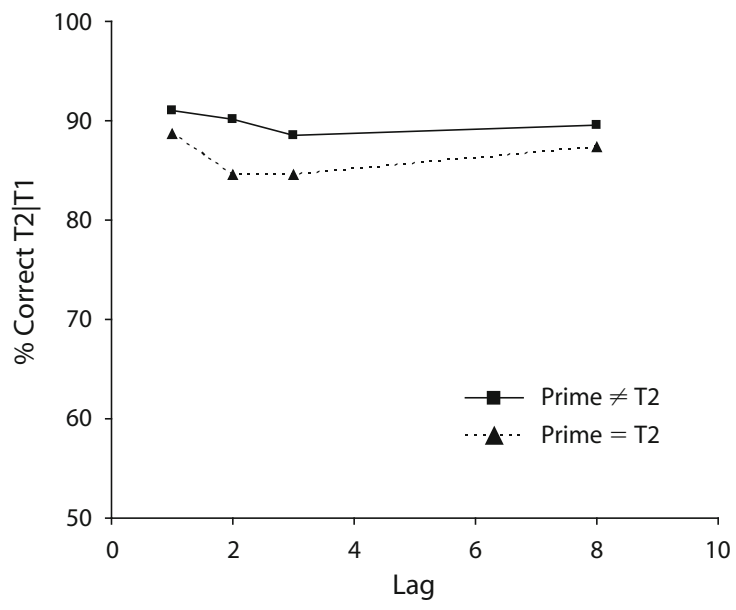

Figure 6. Results of Experiment 5, in percentages correct, for $T 2$ given $T 1$ correct $(T 2 \mid T 1)$ as a function of lag and prime.

ceded by an auditory prime. If an auditory prime were to influence $\mathrm{T} 2$ performance, it could mean that interference takes place between the auditory and visual modalities at an amodal level. If this effect were to yield findings similar to those of Experiments 1 and 2, it would provide additional information concerning the level at which the inhibition we have observed takes place.

\section{Method}

The present experiment was identical to Experiment 1, except that an auditory prime was presented. The prime was a spoken number, delivered in Dutch in a male voice, and was presented by means of Sennheiser HD 202 headphones. The sound samples used had an average duration of $425 \mathrm{msec}$ ( $16 \mathrm{bits}, 44 \mathrm{kHz}$ ). The onset of the sound sample was time-locked $1,000 \mathrm{msec}$ before the onset of the fixation cross. The sound samples were manually normalized for amplitude. Twenty-four new students ( 10 female, 14 male; mean age 20.6 years, age range 15-36) participated in the experiment. All had normal hearing and vision.

\section{Results}

T1 accuracy. T1 was correctly identified on $91 \%$ of the trials. For the prime $=\mathrm{T} 2$ condition, the performance on lags $1,2,3$, and 8 was $88 \%, 94 \%, 94 \%$, and $97 \%$, respectively. In the same order, the results for the prime $\neq$ T2 condition were $87 \%, 94 \%, 95 \%$, and $97 \%$. There was no significant main effect of prime $(F<1)$. The two-way interaction between prime and lag failed to reach significance $(F<1)$, but there was a main effect of $\operatorname{lag}[F(3,69)=$ $\left.28.474, M S_{\mathrm{e}}=.004, p<.001\right]$. Further analysis by means of a two-tailed paired-samples $t$ test showed a significant performance drop on lag 1 as compared with lag $2[t(23)=$ $4.459, p<.001]$ and an increase in performance on lag 8 as compared with lag $3[t(23)=3.551, p<.005]$.

T2 accuracy. The average scores for the individual conditions are shown in Figure 7. The performance on $\mathrm{T} 2$ given $\mathrm{T} 1$ correct varied with lag $[F(3,69)=14.534$, $\left.M S_{\mathrm{e}}=.009, p<.001\right]$, indicating an AB effect. For T2, there was no significant main effect of prime $[F(1,23)=$ $\left.2.461, M S_{\mathrm{e}}=.003, p=.130\right]$. The two-way interaction 
between prime and lag was also not significant $[F(3,69)=$ $\left.1,661, M S_{\mathrm{e}}=.003, p=.204\right]$.

\section{Discussion}

Unlike in Experiments 1 and 2, the present results do not show a significant effect of the prime on T2 performance. A reason for the observed null result could be that cross-modal priming is simply not possible within this paradigm. Even though earlier reports have shown cross-modal priming (Graf et al., 1985) and cross-modal NP (Buchner et al., 2003), in these studies the stimuli were words and line drawings. In order to find a crossmodal effect, input from different sensory modalities might need to interact on a level at which amodal semantic representations have already formed. In line with the results of our Experiment 3, the present study confirms that priming at a semantic level does not cause a negative effect on $\mathrm{T} 2$ performance.

\section{GENERAL DISCUSSION}

This study began with the premise that in an AB task, priming $\mathrm{T} 2$ with an identical item has an inhibitory effect on T2 performance. Both Experiments 1 and 2 showed this effect and revealed that this effect adds to the classic AB effect. Similar effects were reported in earlier studies (Akyürek \& Hommel, 2005; Nieuwenstein et al., 2007). One of our conjectures was that this effect might be the result of NP. If this is indeed the case, one would expect the effect to disappear when T1 was from a different character class than the prime and T2. Indeed, one can argue that when no competition between prime and T1 takes place, there is no need for inhibition. Our Experiment 4 confirmed this prediction by showing that the inhibitory effect of the prime disappears when $\mathrm{T} 1$ is changed. In addition, we wanted to determine whether this inhibitory effect would also occur during unimodal auditory priming and during auditory-visual cross-modal priming. Experiment 5 indeed showed an auditory priming effect when $\mathrm{T} 2$ was also pre-

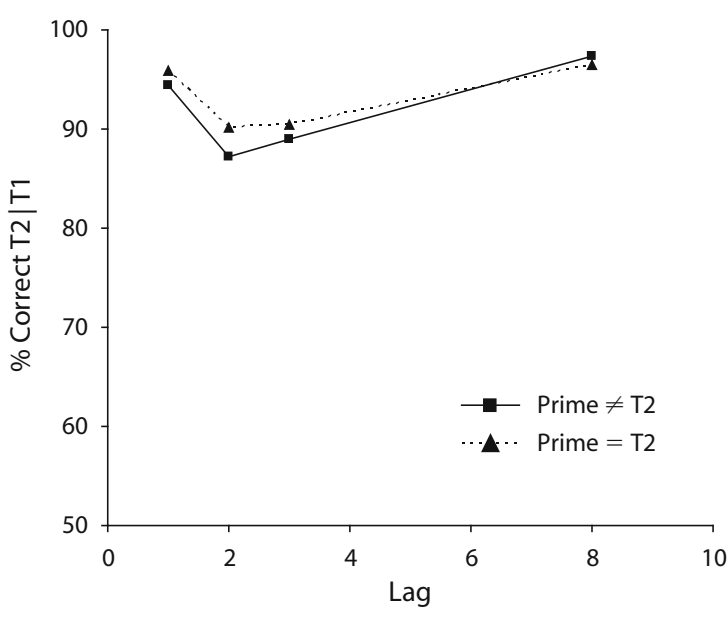

Figure 7. Results of Experiment 6, in percentages correct, for $\mathrm{T} 2$ given $\mathrm{T} 1$ correct $(\mathrm{T} 2 \mid \mathrm{T} 1)$ as a function of lag and prime. sented auditorily. However, cross-modal (auditory-visual; Experiment 6) priming had no effect on T2 performance.

The results of Experiments 1, 2, and 5 are consistent with those from previous studies (Akyürek \& Hommel, 2005; Nieuwenstein et al., 2007) that have shown similar inhibitory effects on T2 performance when this target is preceded by an item that is identical or from a similar character class. The fact that the semantic primes used in Experiments 3 and 6 did not influence T2 performance suggests that the observed inhibition takes place before a semantic level is reached. There are several ways to interpret this unimodal inhibitory priming effect.

The idea that identical items presented in different tasks during the same trial are harder to retrieve than items used in a single task was named "cross-talk repetition amnesia" by Nieuwenstein et al. (2007). In their study, an STM set was presented prior to a standard AB task. When an STM item was identical to one of the $\mathrm{AB}$ targets, performance for these targets decreased. According to the idea of cross-talk repetition amnesia, both the STM item and the targets in the AB task go through a "tokenization" process in which binding between episodic features and item representations takes place. When an item is stored with episodic features from different tasks, retrieval of this item causes interference between these episodic features, and this interference can result in errors during retrieval. Our results indicate that it is not necessary to use a whole STM set; a single prime can also evoke such errors. In other words, no cross-talk between tasks is needed in order to observe inhibition on T2 performance; a mere single item that does not have to be retrieved will have the same effect. One could argue that looking at the prime can be seen as a separate task, in which case the idea of cross-talk repetition amnesia is still valid. In a more general way, our results show that an interaction occurs between items perceived prior to an $\mathrm{AB}$ task and identical items that are part of the RSVP stream, and that this interference does not have to take place during the retrieval period (as the notion of cross-talk repetition amnesia suggests) but could also take place during consolidation of T2. Still, the basic mechanism behind this effect needs to be explained. We reported earlier that the idea of cross-talk repetition amnesia is in line with the episodic retrieval model of NP.

In our Experiment 2, episodic retrieval still cannot be ruled out as a possible explanation. Note that in this experiment the prime was shown for $1.5 \mathrm{sec}$ and that the participants sometimes had to report it. In these prime recall trials, the response to the prime differed from the responses to the targets (T1 and T2) in the other trials. For the prime, participants had to indicate whether or not the prime was similar to a test number, whereas for a target they had to indicate whether it was an odd or even digit. In line with Neill and Mathis (1998), one could argue that the episodic retrieval model holds for this experiment, assuming that the prime and the targets were processed differently; even though both prime and targets would have been stored with a "respond to" tag, the different types of response ("same as" rather than "odd" or "even") would have been stored as well. This difference could explain the NP effect that we found. However, Experiment 4 showed no NP effect when T1 was from a different character class than the prime and T2. According to 
the episodic retrieval model, NP should still have occurred in this case, because the prime and $\mathrm{T} 2$ required different responses. This means that the episodic retrieval model can offer only a partial explanation of our results.

According to the inhibition model, on the other hand, NP seems to operate as a postselection mechanism on a central semantic level and can be influenced by participants' strategies (see May, Kane, \& Hasher, 1995, for a review). Both auditory and visual, as well as cross-modal, priming can occur (Buchner et al., 2003), making this account a possible explanation for the inhibitory effects of unimodal priming observed on T2 performance (Experiments 1,2, and 5). However, as noted earlier, the results from Experiment 2 were not in line with the idea of the inhibition model of NP, which predicts facilitation effects for actively observed primes. Moreover, the results of Experiment 3 showed no effect of semantic priming, and those of Experiments 1 and 2 indicated that participants' strategies have basically no influence. Nevertheless, we also found evidence that inhibition of the prime underlies the drop in $\mathrm{T} 2$ performance observed in our experiments. In Experiment 4 , we determined whether a prime presented prior to the RSVP stream functioned as a distractor item for T1. When we changed the character class of $\mathrm{T} 1$, the inhibitory effect disappeared. This finding supports the notion that we indeed were seeing an effect of NP. However, in light of the results of our other experiments, we cannot conclude that the inhibition model is able to adequately explain this NP, because the model seems to be based on inhibitory processes that take place at a higher level than those that played a role in the effects observed here.

To summarize, the interference found in this study seems to act on a feature extraction level, rather than on a semantic level as reported in earlier studies. In the present $A B$ task, we found unimodal NP effects on T2 performance in both the auditory and visual modalities, but no cross-modal or semantic influences. For this effect to occur, prime and target needed to be identical, both needed to be presented in the same modality, and the target had to be accompanied by another target from the same character class.

\section{AUTHOR NOTE}

This research was funded by support from the Dutch Technology Foundation (STW, Grant VET.7079), the Applied Science Division of the Netherlands Organisation for Scientific Research (NWO), and the Technology Program of the Ministry of Economic Affairs to J.T. and A.B. Correspondence concerning this article should be addressed to T. Koelewijn, Department of Cognitive Psychology, Vrije Universiteit Amsterdam, Boechorststraat 1, 1081BT Amsterdam, The Netherlands (e-mail: t.koelewijn@psy.vu.nl).

\section{REFERENCES}

Aкyürek, E. G., \& Hommel, B. (2005). Short-term memory and the attentional blink: Capacity versus content. Memory \& Cognition, 33, 654-663.

ARnell, K. M., \& Jenkins, R. (2004). Revisiting within-modality and cross-modality attentional blinks: Effects of target-distractor similarity. Perception \& Psychophysics, 66, 1147-1161.

ArNell, K. M., \& Jolicceur, P. (1999). The attentional blink across stimulus modalities: Evidence for central processing limitations. Journal of Experimental Psychology: Human Perception \& Performance, 25, 630-648.
Broadbent, D. E., \& Broadbent, M. H. P. (1987). From detection to identification: Response to multiple targets in rapid serial visual presentation. Perception \& Psychophysics, 42, 105-113.

Buchner, A., ZABAL, A., \& MaYR, S. (2003). Auditory, visual, and crossmodal negative priming. Psychonomic Bulletin \& Review, 10, 917-923.

Chun, M. M., \& PotTer, M. C. (1995). A two-stage model for multiple target detection in rapid serial visual presentation. Journal of Experimental Psychology: Human Perception \& Performance, 21, 109-127.

DeSchepper, B., \& Treisman, A. (1996). Visual memory for novel shapes: Implicit coding without attention. Journal of Experimental Psychology: Learning, Memory, \& Cognition, 22, 27-47.

Graf, P., Shimamura, A. P., \& Squire, L. R. (1985). Priming across modalities and priming across category levels: Extending the domain of preserved function in amnesia. Journal of Experimental Psychology: Learning, Memory, \& Cognition, 11, 386-396.

Houghton, G., Tipper, S. P., Weaver, B., \& Shore, D. I. (1996). Inhibition and interference in selective attention: Some tests of a neural network model. Visual Cognition, 3, 119-164.

JACOBY, L. L., \& DaLlas, M. (1981). On the relationship between autobiographical memory and perceptual learning. Journal of Experimental Psychology: General, 110, 306-340.

Joliceur, P., \& Dell'AcQua, R. (1998). The demonstration of shortterm consolidation. Cognitive Psychology, 36, 138-202.

Martens, S., Munneke, J., Smid, H., \& Johnson, A. (2006). Quick minds don't blink: Electrophysiological correlates of individual differences in attentional selection. Journal of Cognitive Neuroscience, 18, $1423-1438$

May, C. P., Kane, M. J., \& Hasher, L. (1995). Determinants of negative priming. Psychological Bulletin, 118, 35-54.

NeILl, W. T., \& Mathis, K. M. (1998). Transfer-inappropriate processing: Negative priming and related phenomena. In D. L. Medin (Ed.), The psychology of learning and motivation: Advances in research and theory (Vol. 38, pp. 1-44). San Diego: Academic Press.

Neill, W. T., Valdes, L. A., Terry, K. M., \& Gorfein, D. S. (1992). Persistence of negative priming: II. Evidence for episodic trace retrieval. Journal of Experimental Psychology: Learning, Memory, \& Cognition, 18, 993-1000.

Nieuwenstein, M. R., Johnson, A., Kanai, R., \& Martens, S. (2007). Cross-task repetition amnesia: Impaired recall of RSVP targets held in memory for a secondary task. Acta Psychologica, 125, 319-333.

Potter, M. C., Chun, M. M., Banks, B. S., \& Muckenhoupt, M. (1998). Two attentional deficits in serial target search: The visual attentional blink and an amodal task-switch deficit. Journal of Experimental Psychology: Learning, Memory, \& Cognition, 24, 979-992.

PotTer, M. C., STAub, A., \& O'ConNor, D. H. (2002). The time course of competition for attention: Attention is initially labile. Journal of Experimental Psychology: Human Perception \& Performance, 28, 1149-1162.

Raymond, J. E., Shapiro, K. L., \& Arnell, K. M. (1992). Temporary suppression of visual processing in an RSVP task: An attentional blink? Journal of Experimental Psychology: Human Perception \& Performance, 18, 849-860.

Rothermund, K., Wentura, D., \& De Houwer, J. (2005). Retrieval of incidental stimulus-response associations as a source of negative priming. Journal of Experimental Psychology: Learning, Memory, \& Cognition, 31, 482-495.

Spence, C., \& Driver, J. (1997). Audiovisual links in exogenous covert spatial orienting. Perception \& Psychophysics, 59, 1-22.

TIPPER, S. P. (1985). The negative priming effect: Inhibitory priming by ignored objects. Quarterly Journal of Experimental Psychology, 37A, 571-590.

Tipper, S. P., Weaver, B., Cameron, S., Brehaut, J. C., \& Bastedo, J. (1991). Inhibitory mechanisms of attention in identification and localization tasks: Time course and disruption. Journal of Experimental Psychology: Learning, Memory, \& Cognition, 17, 681-692.

Wood, T. J., \& MiLliken, B. (1998). Negative priming without ignoring. Psychonomic Bulletin \& Review, 5, 470-475.

YEe, P. L. (1991). Semantic inhibition of ignored words during a figure classification task. Quarterly Journal of Experimental Psychology, 43A, 127-153.

(Manuscript received August 14, 2006; revision accepted for publication November 26, 2007.) 